\title{
Rapid lipid enrichment in $\omega 3$ fatty acids: Cause-to-effect relationships
}

\author{
YVON A. CARPENTIER, SEBASTIEN PELTIER, LAURENCE PORTOIS, \\ ABDULLAH SENER and WILLY J. MALAISSE \\ Laboratories of Experimental Hormonology and Surgery, Brussels Free University, Brussels, Belgium
}

Received November 7, 2007; Accepted December 11, 2007

\begin{abstract}
The bolus intravenous administration of a novel medium-chain triglyceride:fish oil emulsion to second generation rats depleted in long-chain polyunsaturated $\omega 3$ fatty acids was recently found to enrich within $60 \mathrm{~min}$ the content of both plasma and liver lipids in such $\omega 3$ fatty acids, this coinciding with correction of the perturbation in liver triglyceride fatty acid content and profile otherwise prevailing in these rats. The present report draws attention to cause-toeffect relationships between changes in liver phospholipid and triglyceride fatty acid content and/or pattern operative under these experimental conditions.
\end{abstract}

\section{Introduction}

In the two prior reports in this series, rapid changes in the fatty acid content and profile of both plasma and liver lipids were documented $60 \mathrm{~min}$ after the bolus intravenous administration of a novel medium-chain triglyceride:fish oil emulsion to second generation rats depleted in long-chain polyunsaturated $\omega 3$ fatty acids $(1,2)$. In the present study, advantage was taken of the comparison between plasma and liver data to scrutinize possible cause-to-effect relationships between changes in liver phospholipid and triglyceride fatty acid content and/or profile under the same experimental conditions.

\section{Materials and methods}

The Materials and methods of the present study were already defined in the first report in this series (1).

Correspondence to: Professor W.J. Malaisse, Laboratory of Experimental Hormonology, Brussels Free University, 808 Route de Lennik, B-1070 Brussels, Belgium

E-mail:malaisse@ulb.ac.be

Key words: second generation rats depleted in long-chain polyunsaturated $\omega 3$ fatty acids, plasma and liver lipid fatty acid profile, medium-chain triglyceride:fish oil emulsion

\section{Results}

In order to investigate possible cause-to-effect links between the enrichment of hepatic phospholipids in long-chain polyunsaturated $\omega 3$ fatty acids and changes in liver triglyceride fatty acid content and pattern, attention was first concentrated on the $\omega 3$-depleted rats injected with the MCT:FO emulsion.

The enrichment of hepatic phospholipids in $\omega 3$ fatty acids was judged from the weight percentage of $\mathrm{C} 20: 5 \omega 3$ in these phospholipids. The selection of this variable was motivated by the fact that no $\mathrm{C} 20: 5 \omega 3$, as distinct from $\mathrm{C} 22: 5 \omega 3$ or C22:6w3, was detected in the liver phospholipids of the other w3-depleted rats, whether uninjected with a lipid emulsion or injected with the control MCT:OO emulsion. At this point, it might nevertheless be mentioned that, in the FO rats, there was an almost significant positive correlation $(\mathrm{r}=0.637$; $\mathrm{n}=9 ; \mathrm{p}<0.07$ ) between the C20:5 03 and C22:5w3 weight percentages of liver phospholipids, both expressed relative to the mean value found in rats of the same sex, whilst such was not the case $(\mathrm{r}=0.337 ; \mathrm{n}=9 ; \mathrm{p}>0.1)$ for the comparison between the $\mathrm{C} 20: 5 \omega 3$ and $\mathrm{C} 22: 6 \omega 3$ weight percentages of the liver phospholipids of the same rats.

C16:0 weight percentage in liver triglycerides. The weight percentage of $\mathrm{C} 16: 0$ in the liver triglycerides of rats injected with the MCT:FO emulsion was selected as a first variable dramatically affected by such an injection in both male and female rats (2). Such a weight percentage is much lower in the FO rats than in the uninjected $\omega 3$-depleted rats. Unexpectedly and paradoxically, there was in the FO rats, a positive correlation $(\mathrm{r}=0.662 ; \mathrm{n}=9 ; \mathrm{p}<0.06)$ between the weight percentage of $\mathrm{C} 20: 5 \mathrm{\omega} 3$ in liver phospholipids and that of C16:0 in liver triglycerides, as if the enrichment of liver phospholipids by long-chain polyunsaturated $\omega 3$ fatty acid would cause an increase, and not the observed decrease, in the C16:0 weight percentage of liver triglycerides. An alternative explanation for the latter decrease was searched therefore, by comparing the C16:0 weight percentage of plasma and liver triglycerides in the twelve male rats injected with a lipid emulsion. In this case, the expected positive correlation between these two variables was indeed observed ( $\mathrm{r}=0.650$; $\mathrm{n}=12 ; \mathrm{p}<0.03$ ), suggesting that the fall in the $\mathrm{C} 16: 0$ weight percentage of liver triglycerides reflects the hepatic uptake of injected triglycerides, rather than being linked to changes in the $\mathrm{C} 20: 5 \omega 3$ weight percentage of liver phospholipids. If such 
is the case, there remained to account for the above mentioned positive correlation between phospholipid C20:5 23 and triglyceride $\mathrm{C} 16: 0$ weight percentages found in the rats injected with the MCT:FO emulsion. One possible explanation could be that the injection of the MCT:FO emulsion and resulting correction of $\omega 3$-depletion, by restoring a low activity of $\Delta 9$-desaturase, indeed augment the $\mathrm{C} 16: 0$ relative content of liver triglycerides. Such a hypothesis takes into account the fact that the $\mathrm{C} 16: 1 \omega 7 / \mathrm{C} 16: 0$ ratio in plasma triglycerides was not significantly different $(\mathrm{p}>0.6)$ in the male uninjected $\omega 3$-depleted rats $(0.203 \pm 0.021 ; n=6)$ and those injected with either the MCT:OO emulsion $(0.198 \pm 0.022 ; \mathrm{n}=6)$ or MCT:FO emulsion $(0.186 \pm 0.029 ; n=6)$. It is compatible with the observation that, in both male and female rats, the C16:1 $107 /$ $\mathrm{C} 16: 0$ ratio in liver triglycerides is lower in FO rats than in the other $\omega 3$-depleted rats (2). It is strongly supported by the finding of a significant negative correlation ( $r=-0.688 ; n=9$; $\mathrm{p}<0.05)$ in the nine rats injected with the MCT:FO emulsion, between the absolute values for the $\mathrm{C} 22: 6 \omega 3$ weight percent

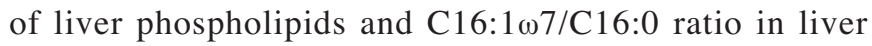
triglycerides (Fig. 1, right panel). In this respect, two remarks should be underlined. First, the significant correlation just mentioned was reached without exclusion of any individual animal and thus included the unfavourable results recorded in one of these animals (2). Second, since such a correlation refers to the absolute values for the two variables under consideration, as recorded in either male or female rats, it suggests that gender differences in the apparent activity of $\Delta 9$-desaturase, as judged for instance from the C16:1 $107 /$ $\mathrm{C} 16: 0$ ratio in liver triglycerides, may also be related to the relative content of C22:6w3 in liver phospholipids. Such a relative content was higher $(\mathrm{p} \leq 0.01)$ in female rats than in male rats, whether injected or not with the MCT:FO emulsion and this coincided with a lower $\mathrm{C} 16: 1 \omega 7 / \mathrm{C} 16: 0$ ratio in liver triglycerides of female versus male rats, whether in the animals injected with the MCT:FO emulsion $(\mathrm{p}<0.04)$ or the other animals $(\mathrm{p}<0.04)$.

As a matter of fact, in the male and female w3-depleted rats, that were either not injected or injected with the control MCT:OO emulsion, there was also a significant inverse correlation $(\mathrm{r}=-0.656 ; \mathrm{n}=14 ; \mathrm{p}<0.02)$ between the absolute values for the $\mathrm{C} 22: 6 \omega 3$ weight percentage in liver phospholipids and the $\mathrm{C} 16: 1 \omega 7 / \mathrm{C} 16: 0$ ratio in liver triglycerides (Fig. 1, left panel). It could obviously be objected that such a correlation merely reflects the gender difference in the two variables under consideration. However, even when considering only the twelve male NI and OO rats and even when expressing all results relative to the mean value found in each of these two groups of male rats, there remained a significant inverse correlation $(\mathrm{r}=-0.608 ; \mathrm{n}=12 ; \mathrm{p}<0.05)$ between the two variables under consideration. This documents that, even within homogeneous group(s) of rats of the same sex and exposed to the same experimental conditions, the $\mathrm{C} 16: 1 \omega 7 / \mathrm{C} 16: 0$ ratio in liver triglycerides tightly depends on the $\mathrm{C} 22: 6 \omega 3$ relative content of liver phospholipids.

Importantly, the covariance analysis of the two sets of data illustrated in Fig. 1 indicated that the slope of the regression lines was not significantly different in the FO rats $(-13.3 ; n=9)$ and in the other animals $(-12.9 ; n=14)$, and that these lines also failed to differ significantly in their elevation

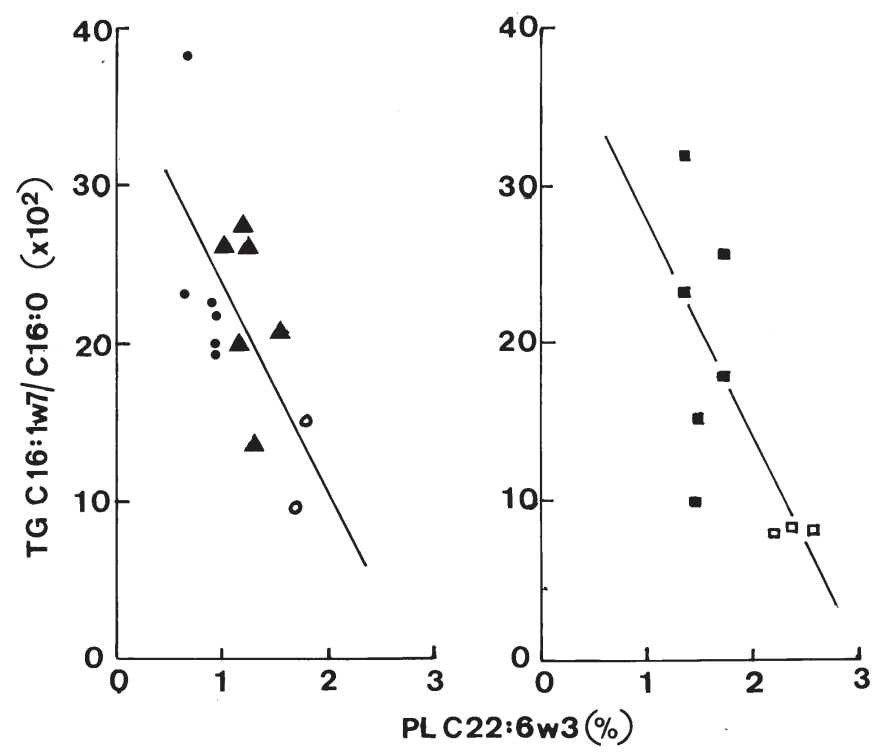

Figure 1. Negative correlation between the absolute values for the $\mathrm{C} 16: 1 \omega 7 / \mathrm{C} 16: 0$ ratio in liver triglycerides and C22:6 13 weight percentage in liver phospholipids of male (black symbols) and female (white symbols) w3-depleted rats, either uninjected (circles) or injected with the control MCT:OO emulsion (triangles), as illustrated in the left panel, and $\omega 3$-depleted rats injected with the MCT:FO emulsion, as documented in the right panel. The oblique line corresponds to the regression line.

$(F=1.1232 ; f=1,20 ; p>0.25)$. In other words, the two sets of data could be considered as belonging to the same single population.

A further support to the cause-to-effect relationship between the $\mathrm{C} 22: 6 \omega 3$ weight percentage in liver phospholipids and $\mathrm{C} 16: 1 \omega 7 / \mathrm{C} 16: 0$ ratio in liver triglycerides is suggested by the fact that, in sixteen normal female rats, examined in a prior investigation (3), the $\mathrm{C} 22: 6 \mathrm{\omega} 3$ relative content in liver phospholipids $(15.2 \pm 0.5 \%)$ was much higher $(\mathrm{p}<0.001)$ than that here found in the $\omega 3$-depleted female rats injected with the MCT:FO emulsion $(2.4 \pm 0.1 \%)$, this coinciding with a C16:1 $1 / \mathrm{C} 16: 0$ ratio in liver triglycerides $(4.55 \pm 0.30 \%)$ much lower $(\mathrm{p}<0.001)$ than that recorded in the present study in the female FO rats $(8.24 \pm 0.11 \%)$.

C18:2 $\omega 6$ and C22:4 66 weight percentage in liver triglycerides. A comparable situation was observed in the case of the longchain polyunsaturated $\omega 6$ fatty acids $C 18: 2 \omega 6$ and $C 22: 4 \omega 6$. As shown in Fig. 2, in the male and female rats injected with the MCT:FO emulsion, the weight percentage of C18:2 $\omega 6$ in the liver triglycerides was inversely related $(r=-0.936$; $n=9$; $\mathrm{p}<0.001$ ) to the $\mathrm{C} 20: 5 \omega 3$ weight percentage in liver phospholipids both expressed relative to the mean value found in animals of the same sex, suggesting a possible cause-to-effect link between changes in the $\omega 3$ fatty acid content of liver phospholipids and resulting changes in the C18:2 $\omega 6$ content of liver triglycerides. Such a proposal contrasts with the finding that, in the male rats injected with the MCT:FO emulsion, the $\mathrm{C} 18: 2 \omega 6$ relative content of liver triglycerides is significantly increased, when compared to that of uninjected animals, despite the opposite change, i.e. a lowering of the C18:2 $\omega 6$ weight percentage, in the plasma triglycerides of the same animals. 


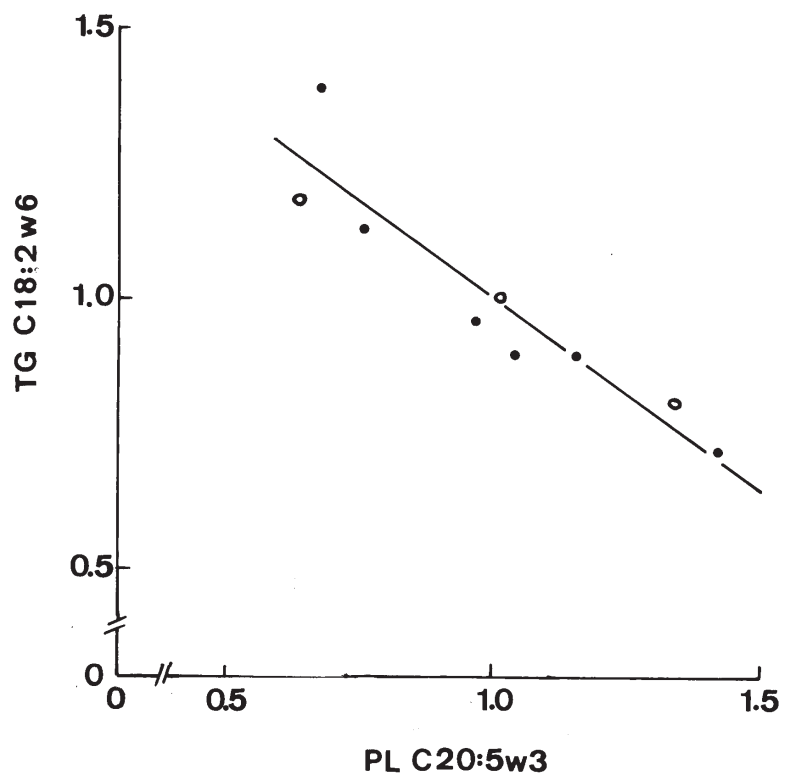

Figure 2. Relationship between the C20:5w3 weight percentage in liver

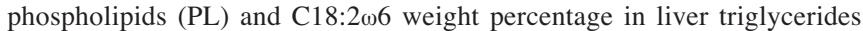
(TG) of male (black circles) and female (white circles) rats injected with the MCT:FO emulsion. Both variables are expressed relative to the mean value found in rats of the same sex. The oblique line represents the regression line.

The analysis of the data collected in the case of C22:4w6 call for somewhat more sophisticated comments. At the first glance, the increase in the weight percentage of C22:4 $\omega 6$ in the triglycerides of FO rats, as observed in both male and female rats could not be blamed on the uptake of plasma triglycerides with a high weight percentage in this long-chain polyunsaturated $\omega 6$ fatty acids since the relative abundance of C22:4 6 in plasma triglycerides was lower, albeit not significantly so, in the rats injected with a lipid emulsion than in the uninjected male $\omega 3$-depleted rats (1). In this respect, it could be argued that, in the former rats, the weight percentage of C22:4 106 was virtually identical in plasma and liver triglycerides $(0.57 \pm 0.07$ and $0.56 \pm 0.08 \%$, respectively; $n=12)$, with a positive correlation $(\mathrm{r}=0.684 ; \mathrm{n}=12 ; \mathrm{p}<0.02)$ between the two variables, whilst it was about thrice higher $(\mathrm{p}<0.025$; paired comparison) in plasma $(0.96 \pm 0.28)$ than in liver $(0.32 \pm 0.13)$ in the six uninjected male $\omega 3$-depleted rats. Hence, the proposal could be made that, at variance with the situation found in uninjected rats, the plasma and liver triglycerides yield the same fatty acid profile after injection of a lipid emulsion. The bulk of the present data indicate, however, the inconsistence of such a proposal. An alternative hypothesis could be that the increase in the weight percentage of C22:4 06 in the triglycerides of FO rats would be causally related to the increase in the long-chain polyunsaturated $\omega 3$ fatty acid content of liver phospholipids. However, in the male and female rats injected with the MCT:FO emulsion, there was again a significant negative correlation $(r=-0.680 ; n=9$; $\mathrm{p}<0.005)$ between the $\mathrm{C} 20: 5 \omega 3$ weight percentage of liver phospholipids and the $\mathrm{C} 22: 4 \omega 6$ weight percentage of liver triglycerides, both expressed relative to the mean value found in animals of the same sex. At the first glance, the latter finding also strongly argued against this second hypothesis. It was then considered, as a third hypothesis, that the injection of

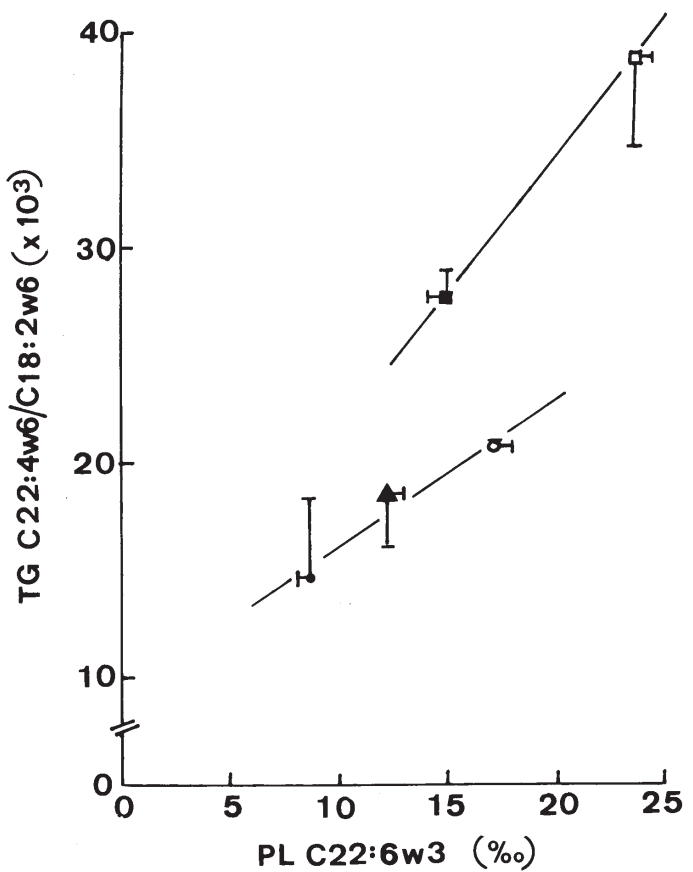

Figure 3. Relationship between the absolute values for the $\mathrm{C} 22: 6 \omega 3$ weight percentages of liver phospholipids and C22:4 $\omega 6 / \mathrm{C} 18: 2 \omega 6$ ratio (geometric means) in liver triglycerides of uninjected female (white circles) and male (black circles) rats, as well as male rats injected with the MCT:OO emulsion (black triangles), and female (white squares) and male (black squares) animals injected with the MCT:FO emulsion. The oblique lines refer to the regression lines, as computed from the mean values.

the MCT:FO emulsion, by raising the liver phospholipid content in $\omega 3$ fatty acids, may somehow favour the stepwise conversion of $\mathrm{C} 18: 2 \omega 6$ to $\mathrm{C} 22: 4 \omega 6$. This proposal is consistent with the much higher $\mathrm{C} 22: 4 \omega 6 / \mathrm{C} 18: 2 \omega 6$ ratio found in the liver triglycerides of both male and female FO rats, as compared to either uninjected animals or OO rats of the same sex (2). In the male and female rats injected with the MCT:FO emulsion, the positive correlation between the C20:5 33 weight percentage of liver phospholipids and C22:4 66 / C18:2w6 ratio in liver triglycerides failed to achieve statistical significance $(p>0.1)$, when the results were expressed relative to the mean value found in rats of the same sex. However, in the same nine rats, there was a positive correlation $(\mathrm{r}=0.752 ; \mathrm{n}=9 ; \mathrm{p}<0.03)$ between the absolute values for the C22:6w3 weight percentage of liver phospholipids and $\mathrm{C} 22: 4 \omega 6 / \mathrm{C} 18: 2 \omega 6$ ratio in liver triglycerides (Fig. 3). The latter finding not only affords support to the existence of a causeto-effect link between the enrichment of liver phospholipid in long-chain polyunsaturated $\omega 3$ fatty acid, as resulting from the injection of the MCT:FO emulsion, and the observed increase in the triglyceride $\mathrm{C} 22: 4 \omega 6 / \mathrm{C} 18: 2 \omega 6$ ratio. It may also shed light on the mechanism(s) underlying gender differences in the latter variable. And, indeed, not only in the FO rats but also in the uninjected rats and those injected with the control MCT:OO emulsion, the higher C22:6w3 content of liver phospholipids found in the female animals coincided with a mean higher $\mathrm{C} 22: 4 \omega 6 / \mathrm{C} 18: 2 \omega 6$ ratio in liver triglycerides than in male animals (2). Pooling together the two sets of data, the $\mathrm{C} 22: 4 \omega 6 / \mathrm{C} 18: 2 \omega 6$ ratio in liver triglycerides averaged, in male rats, no more than $70.9 \pm 6.9 \%(n=10 ; p<0.02)$ 


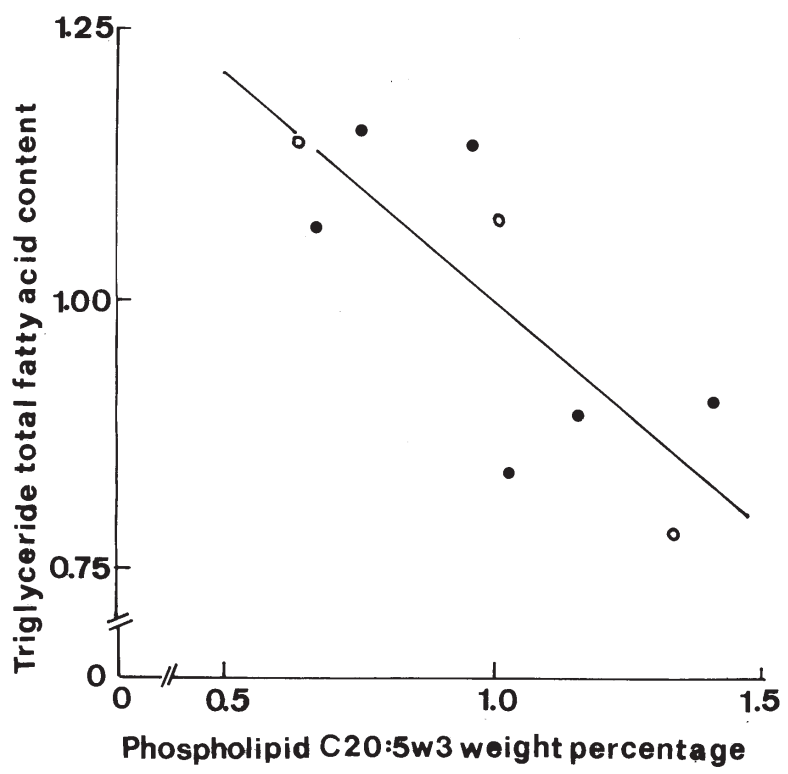

Figure 4. Correlation between the triglyceride total fatty acid content and phospholipid C20:5 03 weight percentage in the liver of male (black circles) and female (white circles) w3-depleted rats injected with the MCT:FO emulsion. Both variables are expressed relative to the mean value found in rats of the same sex. The oblique line corresponds to the regression line.

of the mean value $(100.0 \pm 5.0 \% ; \mathrm{n}=5)$ found in female rats treated in the same manner in terms of the exogenous supply of long-chain polyunsaturated $\omega 3$ fatty acids. As illustrated in Fig. 3, the male rats injected with the MCT:OO emulsion occupied a position in between those recorded in the male and female uninjected animals, reinforcing the concept of a link between the $\mathrm{C} 22: 6 \omega 3$ content of liver phospholipids and C22:4 $\omega 6 / \mathrm{C} 18: 2 \omega 6$ ratio in liver triglycerides.

In the FO rats, the $\mathrm{C} 22: 6 \omega 3$ weight percentage in liver phospholipids remained much lower than that recorded in prior investigations conducted in normal rats $(3,4)$. Such was the case in both male and female rats with mean respective values of $1.50 \pm 0.07(n=6)$ and $2.36 \pm 0.10(n=3)$ in the $\omega 3$ depleted rats injected with the MCT:FO emulsion, as distinct $(\mathrm{p}<0.001)$ from $13.01 \pm 1.19(\mathrm{n}=5)$ and $15.16 \pm 0.45(\mathrm{n}=16)$ in the normal animals. Incidentally, these data reveal that, in normal rats like in $\omega 3$-depleted animals, such a phospholipid $\mathrm{C} 22: 6 \omega 3$ relative content remained lower $(\mathrm{p}=0.05)$ in males than in females. Despite the dramatic difference in C22:6 33 content of liver phospholipids in $\omega 3$-depleted rats injected with

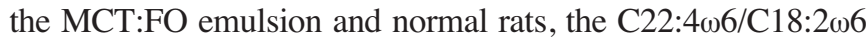
ratio in liver triglycerides failed to differ significantly, whether in male or female, when comparing the former animals to the latter rats. Such a ratio indeed averaged, in the FO and control animals respectively, $27.9 \pm 1.2$ and $23.5 \pm 2.9 \times 10^{-3}(\mathrm{p}>0.17)$ in male rats and $39.2 \pm 4.0$ and $32.4 \pm 2.0 \times 10^{-3}(\mathrm{p}>0.19)$ in female rats. It was significantly higher in females than in males, whether in the $\omega 3$-depleted rats injected with the MCT:FO emulsion $(p<0.01)$ or control animals $(p<0.04)$. At the first glance, these findings extent to normal rats the concept that the gender differences in the $\mathrm{C} 22: 6 \omega 3$ content of liver phospholipids accounts for the gender difference in the C22:4 $66 /$ C18:2 $\omega 6$ ratio of liver triglycerides. However, in the normal female rats, an unexpected negative correlation $(\mathrm{r}=-0.646$; $\mathrm{n}=16 ; \mathrm{p}<0.01)$ was found between these two variables. Even in a small group of five normal male rats, a significant negative correlation between the same variables was also observed $(\mathrm{r}=-0.951 ; \mathrm{n}=5 ; \mathrm{p}<0.02)$. Taken as a whole, these findings call for nuanced comments. First, it should not be ignored that, at variance with the situation found in both normal and $\omega 3$-depleted uninjected rats, that prevailing in the $\omega 3$-depleted rats injected with the MCT:FO emulsion may not refer to a long-term steady-state picture. Second, it could be argued that the value for the $\mathrm{C} 22: 6 \omega 3$ content of liver phospholipids reached in the latter rats is already sufficient to ensure an optimal regulation of the stepwise conversion of C18:2 $\omega 6$ to $\mathrm{C} 22: 4 \omega 6$ and their incorporation in liver triglycerides. Last, despite the analogy between $\omega 3$-depleted rats and normal animals in terms of the apparent link between the C22:6 13 content of liver phospholipids and C22:4 $\omega 6 / \mathrm{C} 18: 2 \omega 6$ ratio of liver triglycerides, the unexpected finding of a negative correlation between the individual values for these two variables in both male and female normal rats strongly suggests that, in these rats, other factor(s) than the C22:6w3 content of liver phospholipids participate(s) in the fine regulation of long-chain polyunsaturated $\omega 6$ fatty acid metabolism in hepatocytes.

Total fatty acid content of liver triglycerides. The last, but not least important, significant correlation $(\mathrm{r}=-0.7801 ; \mathrm{n}=9$; $\mathrm{p}<0.02$ ) between the weight percentage of $\mathrm{C} 20: 5 \mathrm{w} 3$ in liver phospholipids and a variable of liver triglycerides concerned their total fatty acid content in male and female $\omega 3$-depleted rats injected with the MCT:FO emulsion. As shown in Fig. 4, this consisted in an inverse relationship, as if the relative extent of the phospholipid enrichment in long-chain polyunsaturated $\omega 3$ fatty acids provoked by the prior intravenous injection of the MCT:FO emulsion to the $\omega 3$-depleted rats dictated the relative extent of the lowering in liver triglyceride content. A partial correction of liver steatosis, which otherwise prevails in the $\omega 3$-depleted rats, was first documented in male w3-depleted rats injected $60 \mathrm{~min}$ before liver sampling with the MCT:FO emulsion (4).

\section{Discussion}

The major contribution of this study consists in the identification of cause-to-effect relationships in response to the bolus intravenous injection of the MCT:FO emulsion to w3-depleted rats. In such a perspective, attention was focused on the links between the enrichment of liver phospholipids in long-chain polyunsaturated $\omega 3$ fatty acids and the resulting changes in the fatty acid content and pattern of liver triglycerides. In this respect, the two following sets of findings are quite illustrative.

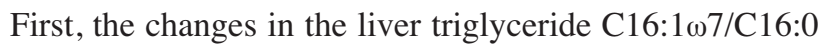
and $\mathrm{C} 22: 4 \omega 6 / \mathrm{C} 18: 2 \omega 6$ ratios provoked by the prior injection of the MCT:FO emulsion could not be blamed on the modification of these ratios in circulating triglycerides and, instead, were closely related with the enrichment of liver phospholipids in C22:6w3 (Figs. 1 and 3). These close correlations between the liver triglyceride and phospholipid data may also account, in part at least, for gender differences in the lipid variables under consideration. In the rats injected with the MCT:FO 
emulsion, there was also a significant negative correlation between the liver triglyceride $\mathrm{C} 18: 1 \omega 9 / \mathrm{C} 18: 0$ ratio and C20:5 03 weight percentage of liver phospholipids.

Second, in the rats injected with the MCT:FO emulsion, there was once again a significant negative correlation between the total fatty acid content of liver triglycerides and C20:5 w3 weight percentage of liver phospholipids (Fig. 4). In this respect, the biological significance of the decrease in the triglyceride liver content provoked by the prior injection of the MCT:FO emulsion is reinforced by the consideration that the intravenously injected triglycerides were, in part at least, cleared from circulation through their hepatic uptake, as documented by a number of convergent findings in this study. The latter situation is reminiscent of that previously documented in endothelial cells exposed in vitro to increasing concentrations of the MCT:FO emulsion (5).

\section{Acknowledgements}

This study was supported by a grant (3.4574.07) from the Belgian Foundation for Scientific Medical Research. We are grateful to A. Chwalik and A. Dufour for technical assistance, and to C. Demesmaeker for secretarial help.

\section{References}

1. Carpentier YA, Peltier S, Portois L, Sener A and Malaisse WJ: Rapid lipid enrichment in $\omega 3$ fatty acids: Plasma data. Int J Mol Med 21: 355-365, 2008.

2. Carpentier YA, Peltier S, Portois L, Sener A and Malaisse WJ: Rapid lipid enrichment in $\omega 3$ fatty acids: Liver data. Int J Mol Med 21: 367-373, 2008.

3. Louchami K, Zhang Y, Oguzhan B, Delporte C, Portois L, Carpentier YA, Genten F, Danguy A, Malaisse WJ and Sener A: Rapid changes in liver lipid composition and pancreatic $\mathrm{K}^{+}$ handling and secretory behaviour provoked by the intravenous administration of a medium-chain triglyceride:fish oil emulsion to long-chain polyunsaturated $\omega 3$ fatty acid-depleted rats. Int J Mol Med 18: 1047-1055, 2006.

4. Portois L, Peltier S, Chardigny JM, Sébédio JL, Leverve X, Malaisse WJ and Carpentier YA: Rapid incorporation of longchain polyunsaturated $\omega 3$ fatty acids and lowering of triglycerides in the liver of 133 -depleted rats injected with a medium-chain triglyceride-fish oil emulsion. Eur J Physiol 450: R11, 2005.

5. Carpentier YA, Dupont I, Portois L and Malaisse WJ: Preclinical investigations of a medium-chain triglyceride:fish oil emulsion. III. Experiments in cultured endothelial cells. Int $\mathbf{J}$ Mol Med 18: 1177-1185, 2006. 